\title{
Lumbar disc reherniation after transforaminal lumbar endoscopic discectomy
}

\author{
Thomas A. Kosztowski, David Choi, Jared Fridley, Michael Galgano, Ziya Gokaslan, Adetokunbo \\ Oyelese, Albert Edward Telfeian
}

The Warren Alpert Medical School of Brown University, Providence, Rhode Island, USA

Contributions: (I) Conception and design: All authors; (II) Administrative support: All authors; (III) Provision of study materials or patients: All authors; (IV) Collection and assembly of data: All authors; (V) Data analysis and interpretation: All authors; (VI) Manuscript writing: All authors; (VII) Final approval of manuscript: All authors.

Correspondence to: Albert Edward Telfeian. The Warren Alpert Medical School of Brown University, Providence, Rhode Island, USA.

Email: atelfeian@lifespan.org.

Background: Transforaminal lumbar endoscopic discectomy is a minimally invasive surgical procedure that can be performed in awake patients through an incision less than $1 \mathrm{~cm}$. The procedure requires very little bony removal to access the herniated disc material because the approach is through the foramen, and only a small amount of the superior articulating process is removed to access Kambin's triangle. This study describes our experience with transforaminal endoscopic lumbar decompression (TELD) for the treatment of lumbar disc herniation. We evaluate the risk for reherniation in the first year after surgery and the characteristics of the patients who experience reherniation.

Methods: We describe the technique for the transforaminal endoscopic approach to treat lumbar disc herniations. Retrospectively, a series of 141 consecutive patients, who were operated on with lumbar radiculopathy, was analyzed. We excluded patients who had previous surgery at the lumbar level, surgeries done for disease adjacent to a fusion, and surgeries done for spondylolisthesis. A total of 84 consecutive patients were included who had single level lumbar non-revision surgery and at least 1-year follow up.

Results: A series of 46 consecutive male and 38 female patients with an average age of 57.4 years (range, 28-87 years old) who underwent transforaminal endoscopic treatment for lumbar disc herniations between 2014 and 2016 is presented. Four patients required microdiscectomy due to reherniation at 5 months, 8 months, 9 months, and 10 months postoperatively. All the patients in the series improved immediately following their endoscopic procedures, and no patients presented with symptoms suggestive of reherniation until 5 months after their initial endoscopic surgery. Patients with reherniation tended to be young: 31,45 , 48 , and 49 years of age: all less than the average patient age who underwent endoscopic surgery.

Conclusions: Transforaminal endoscopic surgical access to lumbar disc herniations is an ultra-minimally invasive approach for the treatment lumbar degenerative disc disease. It allows for neural decompression by removing disc and foraminal pathology with minimal bone removal. This minimal bone removal prevents iatrogenic destabilization. However, the 1 -year reherniation rate presented here is $4.7 \%$. This suggests that the benefit of this technique may be that it is ultra-minimally invasive, but it may only be equal, not superior to microdiscectomy in its rate of reherniation.

Keywords: Reherniation; lumbar disc herniation; endoscopic spine surgery; TESSYS; transforaminal

Submitted Jan 11, 2018. Accepted for publication Feb 25, 2018.

doi: $10.21037 /$ atm.2018.02.26

View this article at: http://dx.doi.org/10.21037/atm.2018.02.26 


\section{Introduction}

Surgical treatment of disc disease and foraminal pathology has evolved over time from traditional open spine surgery to minimally invasive approaches including transforaminal endoscopic surgery. The traditional open interlaminar approach has been described since the early $20^{\text {th }}$ century (1). In the early 1970s, percutaneous surgical approaches were introduced $(2,3)$. Surgical approaches utilizing a microscope started being utilized in the late 1970s (4). In 1983, Kambin and Gellman introduced the transforaminal endoscopic lumbar discectomy (5). Then, in the 1990s, the full endoscopic approach with posterolateral access was developed (6-9). Lateral access in full endoscopic transforaminal surgery has been performed since the late 1990s (10).

The surgical procedure of transforaminal endoscopic lumbar decompression (TELD) has the benefits of an ultraminimally invasive spine surgery including small incision, faster recovery, less need for pain medications, and lower blood loss while still achieving equally effective pain reduction compared to traditional open surgical techniques (11-15). TELD does not affect spinal stability and forms minimal scar adhesions $(13,16)$. This technique has gained increasing popularity as patient demand for more minimally invasive approaches has grown. Furthermore, advances in endoscopic visualization and instrumentation have contributed to this technique.

\section{Methods}

\section{Patients}

After Institutional Review Board Approval, charts were obtained and reviewed from 84 consecutive patients who underwent transforaminal endoscopic lumbar discectomy between 2014 and 2016. This group consisted of 46 male and 38 female patients with a mean age of 57.4 years. Data on patient characteristics and time to recurrence are presented primarily.

\section{Operative technique}

For positioning, patients were placed prone on a Wilson frame. The surgery was performed utilizing local and intravenous sedation. Anesthetic was titrated to the level that the patient was still able to communicate throughout the surgery. We utilized the Joimax TESSYS endoscopic system was used for the surgery. A 25 -cm 18-gauge needle was percutaneously inserted through the skin $12-16 \mathrm{~cm}$ lateral to the midline of the back. Intermittent fluoroscopy was used, alternating between lateral and anterior-posterior (AP) view, to guide the needle through Kambin's triangle and into the disc space. Making sure to avoid the exiting and traversing nerve roots. AP fluoroscopy view was utilized to ensure that the needle was past the medial border of the pedicle before the disc space was entered.

To enlarge the neural foramen, sequential reamers were utilized to remove the ventral aspect of the superior articulating process. To unroof the traversing nerve root, an oblique angle was taken to endoscopically drill the junction between the superior articulating process and the pedicle (Figure 1). Straight-, upgoing-, and bendablegraspers were utilized to perform the discectomy. A 360 -degree visualization of the annulus including the exiting and traversing nerve roots was possible because of the rotating, beveled cannula working channel and endoscope. The foraminotomy procedure complete when there was visualization of the exiting and traversing nerve root and when a ball probed dilator could freely pass under the exiting nerve root and over the pedicle of the caudal level (Figure 1). After adequate decompression was achieved through the discectomy and foraminotomy, the patient was asked prior to terminating the procedure the status of her/his radicular symptoms. Once acceptable results were achieved, the working channel and scope were removed. Pressure was applied on the $5 \mathrm{~mm}$ incision for 5 minutes. Once hemostasis had been obtained, the incision was reapproximated with a single, interrupted suture.

\section{Results}

A series of 84 consecutive patients who underwent TELD between 2014 and 2016 was studied. The minimum followup was 1 year. This group consisted of 46 male and 38 female patients with a mean age of 57.4 years (range, 2887 years).

In regards to the types of disc herniations in the treated group, 25 were far lateral, 59 were paracentral, and none were central disc herniations. In regards to treated levels, the majority were at the L4-5 (32 patients) and L5-S1 (31 patients) disc levels (Table 1). The other levels in the group were L3-4 (16 patients) and L2-3 (5 patients).

Of the 84 patients in the series, 4 patients required a microdiscectomy surgery due to reherniation. The reherniation occurred at $5,8,9$, and 10 months post- 

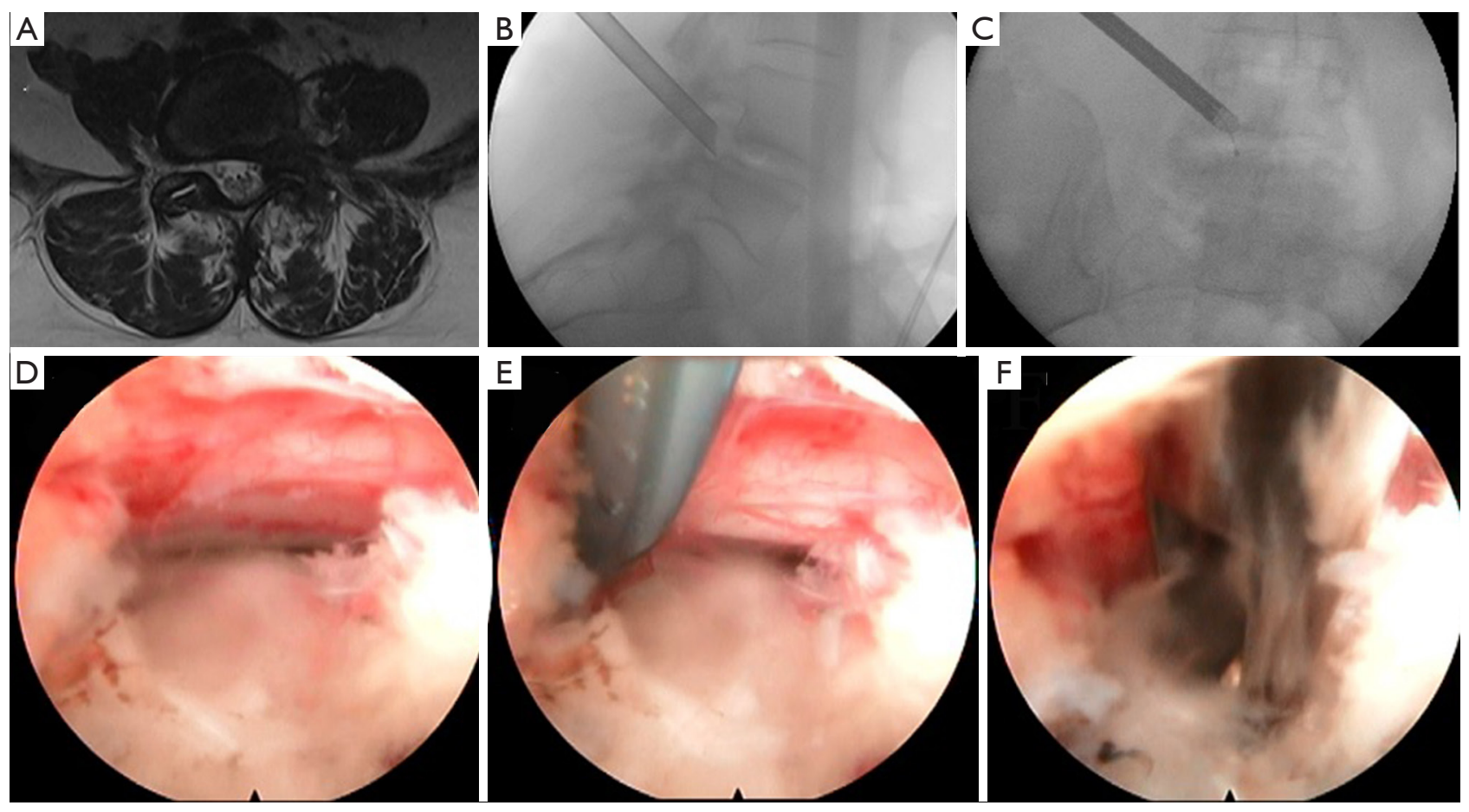

Figure 1 Left lumbar 4-5 foraminal disc herniation in a patient with left L5 radicular symptoms. (A) Axial T2 MRI shows the L4-5 foraminal disc herniation; (B) lateral fluoroscopic view shows the beveled tubular retractor positioned in the L4-5 foramen; (C) AP fluoroscopic view shows the beveled tubular retractor in the left L4-5 foramen with a ball probe over the pedicle of L5; (D) endoscopic view of the L5 nerve root and L4-5 foraminal disc herniation (a portion of the SAP has been removed with reamers to expose the nerve and disc pathology); (E) endoscopic view of the ball probe under the nerve and over the L5 pedicle as pictured in (C); (F) endoscopic view of the Joimax semi-bendable grasper underneath the L5 nerve root removing the disc herniation.

Table 1 Distribution of patients according to disc levels treated

\begin{tabular}{lc}
\hline Level & No. of patients \\
\hline L2-3 & 5 \\
L3-4 & 16 \\
L4-5 & 32 \\
L5-S1 & 31 \\
\hline
\end{tabular}

operatively (Table 2). All four of these patients had improved immediately following their initial endoscopic procedures with no signs of symptoms of residual disc immediately post-operatively. No patients presented with symptoms suggestive of disc reherniation until 5 months after their initial endoscopic surgery. Patients who experienced reherniation tended to be young: 31 , 45,48 , and 49 years of age: all less than the average patient age who underwent endoscopic surgery, 57.4 years old.

\section{Discussion}

Multiple studies in the literature have shown that endoscopic spine surgery is effective in treating lumbar spinal pathologies including lateral, paracentral, central, extruded and contralateral disc herniations in addition to lateral recess stenosis $(12,17,18)$. Several studies have demonstrated that full endoscopic technique discectomies are able to achieve equally effective outcomes as microdiscectomies but with the added benefit of quicker postoperative recovery with lower complication rates reported $(11,19)$.

The overall frequency of same-level recurrent disc herniation requiring reoperation is reported to be about $6 \%$ reported in the literature regardless of the technique (i.e., microdiscectomy or endoscopic techniques) (20). Microdiscectomy reherniation rates range from 2.3-11.8\% in the literature (21). In this series, disc reherniation occurred in 4 of the 84 patients (4.76\%). The recurrent disc herniation rate experienced in this study is similar to other 
Table 2 Characteristics of the patients who experienced disc reherniation

\begin{tabular}{llll}
\hline Age (years) & Gender & Level & Time of reherniation \\
\hline 48 & Male & L4-5 paracentral & 5 months post-op, required microdiscectomy \\
49 & Male & L3-4 far lateral & 10 months post-op, required microdiscectomy \\
31 & Female & L4-5 paracentral & 9 months post-op, required microdiscectomy \\
45 & Male & L5-S1 paracentral & 8 months post-op, required microdiscectomy \\
\hline
\end{tabular}

studies in the literature which have reported reherniation rates of $6-12.5 \%$ after TELD $(22,23)$. Some recent studies assessing the transforaminal endoscopic technique have reported reherniation rates as low as a $0.5 \%(16,24)$.

This study attempts to analyze characteristics of patients who had experienced recurrent disc herniations after TELD. Younger age may contribute to higher chances of recurrence of disc herniation as all the patients who experienced reherniation were younger than 50 years old. This is in concordance with other literature examining reoperation rates in general for all recurrent disc herniations from surgery (25). Our study differs from that of Yao et al. who did a retrospective analysis of 116 patients who had a successful initial percutaneous endoscopic lumbar discectomy via a transforaminal route but who experienced a disc reherniation (26). They concluded that older age ( $\geq 50$ years old), central disc herniation, and obesity (body mass index $\geq 25$ ) contributed to higher risk of disc reherniation (26). Our study, in contrast observed that younger age and paracentral disc reherniation had higher rates of disc recurrence.

Limitations of this study include that it is a small retrospective analysis based on the experience of a single institution. Furthermore, this study analyzes reherniation rates up to one year. Future studies will include larger numbers of patients with longer follow-up available.

\section{Conclusions}

Endoscopic approaches in spine surgery are rapidly gaining attention with a growing amount of literature being published (27). As the instrumentation and visualization continue to improve, endoscopic spine surgery is expected to be utilized at an increasing rate (28). This study represents a retrospective analysis of reherniation rates in transforaminal endoscopic discectomies and the characteristics of those patients who experienced them. Younger patients appeared to be more likely to experience disc reherniation requiring a microdiscectomy. Furthermore, there was a trend for paracentral discs to be more likely than far lateral discs for reherniation.

\section{Acknowledgements}

None.

\section{Footnote}

Conflicts of Interest: The authors have no conflicts of interest to declare.

Etbical Statement: After Institutional Review Board Approval, charts were obtained and reviewed from 84 consecutive patients who underwent transforaminal endoscopic lumbar discectomy between 2014 and 2016.

\section{References}

1. Mixter WJ, Barr JS. Rupture of the intervertebral disc with involvement of the spinal canal. N Engl J Med 1934;211:210-5.

2. Hijikata S, Yamagishi M, Nakayma T. Percutaneous discectomy: a new treatment method for lumbar disc herniation. J Toden Hosp 1975;5:5-13.

3. Maroon JC, G Onik G, Sternau L. Percutaneous automated discectomy: a new approach to lumbar surgery. Clin Orthop Relat Res 1989;238:64-70.

4. Caspar W. A new surgical procedure for lumbar disc herniation causing less tissue damaging through a microsurgical approach. In: Wüllenweber R, Brock M, Hamer J, et al. editors. Lumbar Disc Adult Hydrocephalus. Advances in Neurosurgery, vol 4. Berlin: Springer, 1977.

5. Kambin P, Gellman H. Percutaneous lateral discectomy of the lumbar spine: A preliminary report. Clin Orthop 1983;174:127-32.

6. Kambin P, Casey K, O'Brien E, et al. Transforaminal 
arthroscopic decompression of lateral recess stenosis. J Neurosurg 1996;84:462-7.

7. Kambin P, O'Brien E, Zhou L, et al. Arthroscopic microdiscectomy and selective fragmentectomy. Clin Orthop Relat Res 1998:150-67.

8. Lee SH, Lee SJ, Park KH, et al. Comparison of percutaneous manual and endoscopic laser diskectomy with chemonucleolysis and automated nucleotomy. Orthopade 1996;25:49-55.

9. Mathews HH. Transforaminal endoscopic microdiscectomy. Neurosurg Clin N Am 1996;7:59-63.

10. Ruetten S, Komp M, Godolias G. An extreme lateral access for the surgery of lumbar disc herniations inside the spinal canal using the full-endoscopic uniportal transforaminal approach-technique and prospective results of 463 patients. Spine (Phila Pa 1976) 2005;30:2570-8.

11. Ruetten S, Komp M, Merk H, et al. Recurrent lumbar disc herniation after conventional discectomy: a prospective, randomized study comparing full-endoscopic interlaminar and transforaminal versus microsurgical revision. J Spinal Disord Tech 2009;22:122-9.

12. Ruetten S, Komp M, Merk H, et al. Full-endoscopic interlaminar and transforaminal lumbar discectomy versus conventional microsurgical technique: a prospective, randomized, controlled study. Spine (Phila Pa 1976) 2008;33:931-9.

13. Jasper GP, Francisco GM, Telfeian A. Outpatient, awake, ultra-minimally invasive endoscopic treatment of lumbar disc herniations. R I Med J (2013) 2014;97:47-9.

14. Casimiro M. Short-Term Outcome Comparison Between Full-Endoscopic Interlaminar Approach and Open Minimally Invasive Microsurgical Technique for Treatment of Lumbar Disc Herniation. World Neurosurg 2017;108:894-900.e1.

15. Kim MJ, Lee SH, Jung ES, et al. Targeted percutaneous transforaminal endoscopic diskectomy in 295 patients: comparison with results of microscopic diskectomy. Surg Neurol 2007;68:623-31.

16. Zeng Y, Bao J, Su J, et al. Novel targeted puncture technique for percutaneous transforaminal endoscopic lumbar discectomy reduces X-ray exposure. Exp Ther Med 2017;14:2960-8.

17. Choi G, Lee SH, Lokhande P, et al. Percutaneous endoscopic approach for highly migrated intracanal disc herniations by foraminoplastic technique using rigid working channel endoscope. Spine (Phila Pa 1976) 2008;33:E508-15.

18. Ruetten S, Komp M, Merk H, et al. Surgical treatment for lumbar lateral recess stenosis with the full-endoscopic interlaminar approach versus conventional microsurgical technique: a prospective, randomized, controlled study. J Neurosurg Spine 2009;10:476-85.

19. Li M, Yang H, Yang Q. Full-Endoscopic Technique Discectomy Versus Microendoscopic Discectomy for the Surgical Treatment of Lumbar Disc Herniation. Pain Physician 2015;18:359-63.

20. Parker SL, Mendenhall SK, Godil SS, et al. Incidence of Low Back Pain After Lumbar Discectomy for Herniated Disc and Its Effect on Patient-reported Outcomes. Clin Orthop Relat Res 2015;473:1988-99.

21. Fakouri B, Shetty NR, White TC. Is sequestrectomy a viable alternative to microdiscectomy? A systematic review of the literature. Clin Orthop Relat Res 2015;473:1957-62.

22. Bae JS, Lee SH. Transforaminal full-endoscopic lumbar discectomy in obese patients. Int J Spine Surg 2016;10:18.

23. Sanusi T, Davis J, Nicassio N, et al. Endoscopic lumbar discectomy under local anesthesia may be an alternative to microdiscectomy: A single centre's experience using the far lateral approach. Clin Neurol Neurosurg 2015;139:324-7.

24. Kamson S, Trescot AM, Sampson PD, et al. FullEndoscopic Assisted Lumbar Decompressive Surgery Performed in an Outpatient, Ambulatory Facility: Report of 5 Years of Complications and Risk Factors. Pain Physician 2017;20:E221-31.

25. Abdu RW, Abdu WA, Pearson AM, et al. Reoperation for Recurrent Intervertebral Disc Herniation in the Spine Patient Outcomes Research Trial: Analysis of Rate, Risk Factors, and Outcome. Spine (Phila Pa 1976) 2017;42:1106-14.

26. Yao Y, Liu H, Zhang H, et al. Risk Factors for Recurrent Herniation After Percutaneous Endoscopic Lumbar Discectomy. World Neurosurg 2017;100:1-6.

27. Telfeian AE, Jasper GP, Francisco GM. Transforaminal endoscopic treatment of lumbar radiculopathy after instrumented lumbar spine fusion. Pain Physician 2015;18:179-84.

28. Ruetten S, Komp M, Merk H, et al. Use of newly developed instruments and endoscopes: full-endoscopic resection of lumbar disc herniations via the interlaminar and lateral transforaminal approach. J Neurosurg Spine 2007;6:521-30.

Cite this article as: Kosztowski TA, Choi D, Fridley J, Galgano M, Gokaslan Z, Oyelese A, Telfeian AE. Lumbar disc reherniation after transforaminal lumbar endoscopic discectomy. Ann Transl Med 2018;6(6):106. doi: 10.21037/ atm.2018.02.26 